\title{
Enhancing the Activity of Peroxisome Proliferator- Activated Receptor's (PPAR) Activity through Natural Ligand Binding in Diabetes: Substantial Computational Approach
}

\author{
Lakshmi Huttada1, MB Hiremath ${ }^{1 *}$ and Niketan Laner D'Souza ${ }^{2}$ \\ ${ }^{1} P G$ Department of Studies in Biotechnology and Microbiology, Karnataka University, Dharwad-580 003, Karnataka, India \\ ${ }^{2}$ Sacred Heart Pre-University College, Shimoga-577 201, Karnataka, India
}

\section{Abstract}

Background: Traditional medicines have been potential in regulating blood glucose control, leading to herbal treatment to manage diabetes from long time. Diabetes is proving to be a major problem throughout the world in the recent years. In spite of synthetic medications available in the market, to reduce the ill effects of diabetes and its secondary complications, herbal formulations are preferred due to less side effects and low cost.

Objective: The present study attempts in studying antidiabetic property of an unexploited plant, Exacum pedunculatum, belonging to Gentianaceae family, from the Western Ghats of India.

Methodology: The dried plant material was subjected to extraction for isolating compounds which was purified to obtain biguanide compound and screened in silico to study the antidiabetic nature of the compound.

Result: Promising phytochemicals are present in the extract of the plant. Exacum pedunculatum which was further studied in silico for the drug activity. The experiment showed that the bioactive compound isolated from the selected plant showed higher activity than the drug molecule (Metformin) available in the market.

Conclusion: As diabetes is a multifactorial disease leading to several complications, and demands a multiple therapeutic approach; medicinal plants are being looked up once again for the treatment of diabetes. There is a need for conducting clinical research in herbal drugs, developing simple bioassays for biological standardization, pharmacological and toxicological evaluation, and developing various animal models for toxicity and safety evaluation. It is also important to establish mechanism of action of the active components from these plant extracts.

Keywords: Autodock; Diabetes; Exacum pedunculatum; Hexadecanoic acid; Metformin; Molecular docking; Traditional medicine

\section{Introduction}

Diabetes Mellitus is a group of metabolic disorder, characterized by high blood sugar levels. Pancreas either fail to produce enough insulin or the body cells does not respond to the insulin production from the beta cells [1,2]. Type 2 Diabetes (T2D) is a condition of hyperglycaemia characterized by absolute lack of insulin due to breakdown of islet cells in the pancreas. T2D accounts to about $90 \%$ of total cases of diabetes; whereas the other $10 \%$ is due to Type 1 and Gestational diabetes [3-8]. The classic symptoms of T2D are excess thirst, frequent urination and constant hunger.

Initially, diabetes can be managed by physical exercise and adopting dietary changes. Medication may be needed if the blood sugar levels are not lowered. Metformin is known to be first-line choice of drug for treating diabetes [5-9]. Various drugs with different molecular targets and mechanism of action are available for the treatment and management of diabetes. For example:

(i) Insulin, where it binds to the insulin receptor and works on correcting insulin deficiency.

(ii) Sulfonylureas act on ATP-potassium channel to stimulate insulin secretion.

(iii) Biguanide helps in inhibiting hepatic glucose output.

(iv) Thiazolidinediones act on PPAR $\gamma$, increases insulin sensitivity.

Adverse effects of these drugs have made it unsatisfactory in terms of prevention of complications and in restoring the quality of life. Thus, there is a need for new approaches which can treat T2D and restore the quality of life. And one of the desirable approaches to achieve this goal is to identify and isolate natural agents from herbal sources that enhance glucose (nutrient)-dependent insulin secretion [10,11]

The peroxisome proliferator-activated receptors (PPARs) compose a nuclear receptor subfamily that regulate the gene expression of proteins involved in energy, glucose and lipid metabolism, adipocyte proliferation and differentiation and insulin sensitivity [12-14]. PPARs act as cellular sensors that activate transcription in response to the binding of natural or synthetic ligands. Three subtypes, PPARa, PPAR $\gamma$ and PPAR $\beta$ have been identified. All the subtypes share a high level of sequence and structural homology but exhibit differences in tissue expression and physiological function. PPAR $\gamma$ binds to the agonists and changes its confirmation such that it binds with high affinity to co-activators. This binding facilitates remodelling of chromatin and communicates with the cellular transcriptional machinery. As a result, transcriptional initiation is induced and the levels of PPAR-responsive transcripts increase $[14,15]$.

The insulin-sensitizing action of PPAR $\gamma$ agonists results from their ability to lower circulating free fatty acids by decreasing adipocyte lipolysis, regulate the expression of proteins that modulate insulin

*Corresponding author: MB Hiremath, PG Department of Studies in Biotechnology and Microbiology, Karnataka University, Dharwad-580 003, Karnataka, India E-mail: murigendra@gmail.com

Received February 24, 2016; Accepted March 07, 2016; Published March 15, 2016

Citation: Huttada L, Hiremath MB, D'Souza NL (2016) Enhancing the Activity of Peroxisome Proliferator- Activated Receptor's (PPAR) Activity through Natural Ligand Binding in Diabetes: Substantial Computational Approach. Nat Prod Chem Res 4: 213. doi:10.4172/2329-6836.1000213

Copyright: $\odot 2016$ Huttada L, et al. This is an open-access article distributed unde the terms of the Creative Commons Attribution License, which permits unrestricted use, distribution, and reproduction in any medium, provided the original author and source are credited. 
action and serve as adipose remodelling agents that direct lipids away from lipolytic visceral fat depots and into sub-cutaneous fat tissue containing small, insulin-responsive adipocytes. PPAR $\gamma$ agonist is approved for treating T2D as they target adipose tissue and improve insulin sensitivity and act as antidiabetic agent $[12,13]$.

Plants with medicinal properties have been used in treatment and cure of diseases for the welfare of mankind since the dawn of civilization. Nature has bestowed special abilities in plants which help in synthesizing various chemical compounds to help in performing important biological functions and also to help in fighting against the invading predators $[16,17]$. Approximately, 12,000 such compounds have been identified and isolated till date which is less than $10 \%$ of the total number estimated. Herbal medicines are proven to be beneficial in pharmacology as the chemical compounds in the plants show similar effect on human body in a very identical way to the well understood conventional drugs. One of the effective ways to discover future medicines is through Ethno botany which studies the traditional use of plants by humans $[4,17,18]$.

It was more affordable to use herbs to treat diseases among the nonindustrialized societies than purchasing modern pharmaceuticals Asian and African countries using herbal medicines for their primary health care presently range up to $80 \%$ of the total population according to World Health Organization. Less common use in clinical settings in United States and Europe has been gradually increasing as scientific evidence prove their effectiveness of herbal medicines $[17,18]$. Present study provides a thorough assessment of the potential candidate of the plant to ensure the rational use in diabetes management.

\section{Materials and Methods}

The dried and powdered Exacum pedunculatum plant was used to prepare petroleum ether extract which was then subjected to fractionation on silica gel. Preliminary phytochemical screening was performed on the most bioactive fraction obtained from the petroleum ether extract. Standard method was followed for testing the presence of alkaloids, steroids, flavonoids etc [16]. Structural studies confirmed the presence of aliphatic compounds.

The isolated and identified aliphatic compound was subjected for docking studies. The bioinformatics software like PASS PREDICTION, CHEMSKETCH, SPDBV, SWISS online docking and HEX docking were used. The present study was focused on the aliphatic group of drugs showing antidiabetic activity on transmembrane proteins.

The chemical structure and 2D structure of the compound was drawn from ACD/CHEMSKETCH software, an integrated software package from Advanced Chemistry Development Inc. which allows rapid translation into a realistic 3-Dimensional structure. Chemsketch software helps in the generation of 3D structure of the molecule. This software provides an insight of spatial configurations and relationships to molecular properties. It also facilitates to draw other tautomeric forms of the same compound which is useful to understand the physicochemical properties and its interpretation [17-19]. ADMET properties characterize the drug-like activity of the ligand molecules. The prediction of ADME properties leads to the optimization of the given compound and enhancement of the desired properties. The ADME properties of the bioactive compound were evaluated with the software admetSAR developed by Laboratory of Molecular Modelling and Design, Shanghai Key laboratory of New Drug Design, China. The properties are known to influence metabolism, cell permeation and bioavailability.

The docking analysis of bioactive compound with target protein was performed by SWISS online docking and HEX docking software. SWISS PDB viewer was used to study the interaction of the molecules. This application provides user friendly interface allowing analysis of several proteins at the same time. The proteins can be superimposed in order to deduce structural alignments and compare their active sites or any other relevant regions. Amino acid mutations, H-bonds, angles and distances between atoms can also be obtained using this software.

Docking calculations were carried out using Docking Server [20,21]. Gasteiger partial charges were added to the ligand atoms. Non-polar hydrogen atoms were merged, and rotatable bonds were defined. Docking calculations were carried out on Metformin (USAN/INN) protein model. Essential hydrogen atoms, Kollman united atom type charges, and solvation parameters were added with the aid of AutoDock tools [19,22,23]. Affinity (grid) maps of .. Å grid points and $0.375 \AA$ spacing were generated using the Autogrid program [19,22,23]. AutoDock parameter set- and distance-dependent dielectric functions were used in the calculation of the van der Waals and the electrostatic terms, respectively. Docking simulations were performed using the Lamarckian genetic algorithm (LGA) and the Solis \& Wets local search method [21]. Initial position, orientation, and torsions of the ligand molecules were set randomly. All rotatable torsions were released during docking. Each docking experiment was derived from 2 different runs that were set to terminate after a maximum of 250000 energy evaluations. The population size was set to 150 . During the search, a translational step of $0.2 \AA$, and quaternion and torsion steps of 5 were applied.

\section{Results}

Based on the result of the NMR study, the 3D structure was derived using CHEMSKETCH software and the result is given in the (Figure 1) shows the structure of the isolated and identified bioactive compound drawn using Chemsketch software and (Figure 2) is the structural prediction result of the bioactive compound isolated from the plant using software admetSAR developed by Laboratory of Molecular Modelling and Design, Shanghai Key laboratory of New Drug Design, China. This result shows that the compound had good cell permeation, bioavailability and explains the predicted properties of the aliphatic compound in comparison with metformin (Figures 1-6) (Tables 1-3).

\section{Discussion}

Promising phytochemicals are widely distributed in the Gentianaceae family. Animal research has shown anti-inflammatory, analgesic, hepatoprotective and hypoglycaemic activities of the phytochemicals [24-27]. The above study explains the antidiabetic activity of the unexploited species of Gentianaceae family by in silico method.

The predicted properties of the bioactive compound were in the range as predicted by admetSAR software of known oral drugs. They also satisfy the Lipinski's rule of 5 to be considered as drug-like potential.

Binding of naturally obtained compound from the plant extract to the

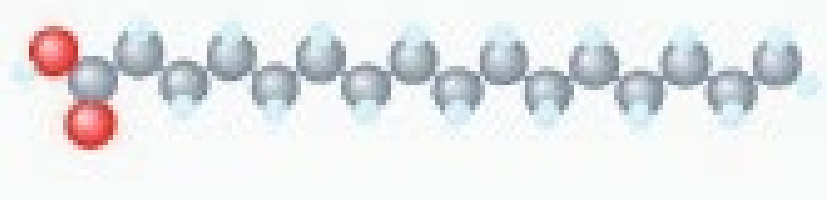

Figure 1: Structure of $n$-Hexadecanoic Acid 


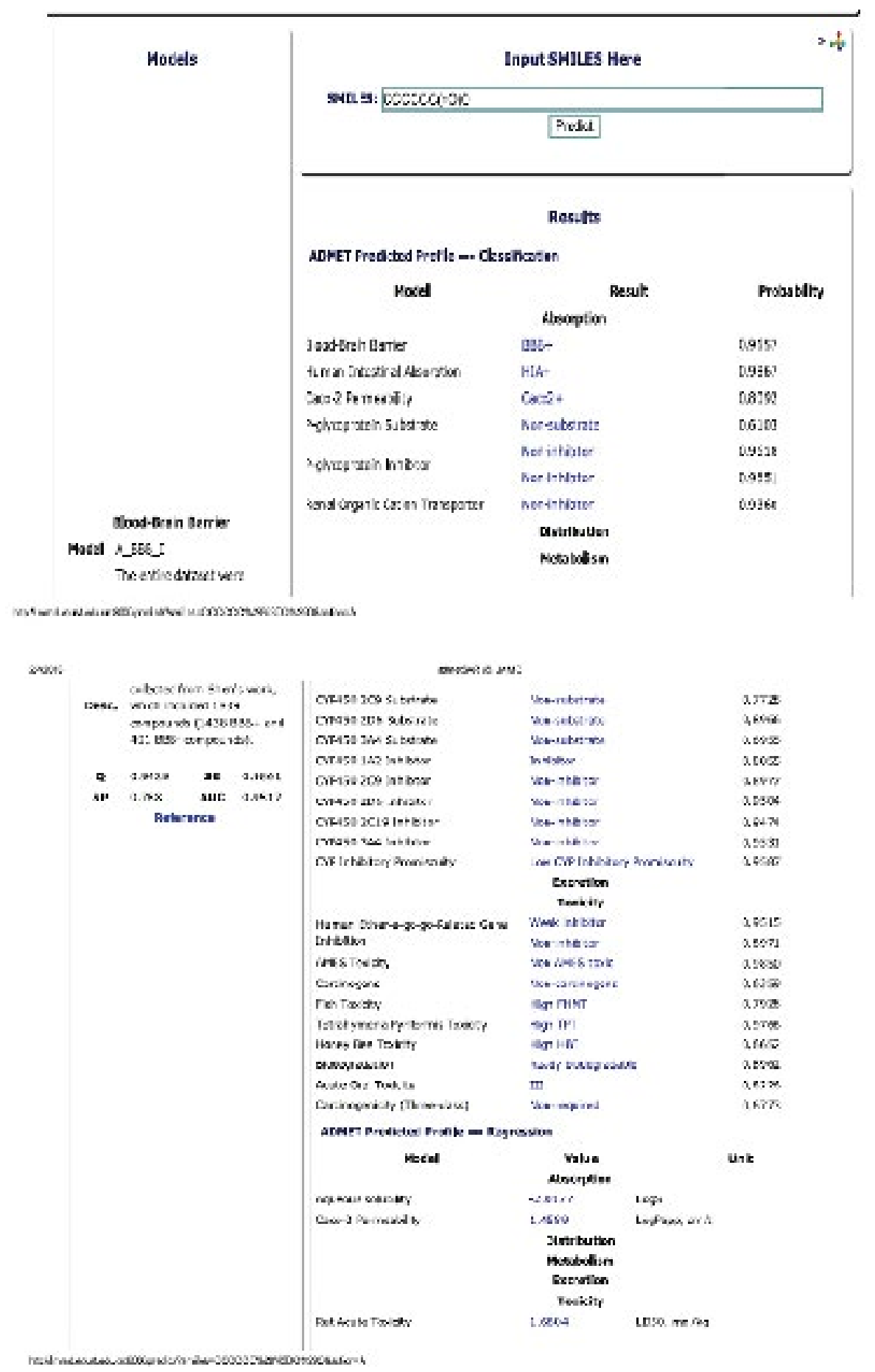

Figure 2: Structural prediction result of the bioactive compound using PASS PREDICTION software.

PPAR $\gamma$ and its expression in the molecular level is studied by virtual screening methods in comparison with the binding and expression of the standard drug Metformin.

Docking studies were carried out for the natural ligand and Metformin as well to understand structural modelling and to predict the activity of specific biological recognition. The process involved the prediction of conformation and orientation of ligand. Interactions between ligand and receptor defined by Van Der Waal's and electrostatic energy terms also support protein-ligand interaction. The free binding energy was higher in the natural ligand $(-5.57 \mathrm{Kcal} / \mathrm{mol})$ compared to the metformin $(-2.69 \mathrm{Kcal} / \mathrm{mol})$, facilitating more active sites for the protein-ligand interaction. As the free binding energy increases, more availability of protein molecules for interaction occurs.

\section{Conclusion}

From the experimental results, we can conclude that naturally obtained bioactive compound has higher activity compared to the 

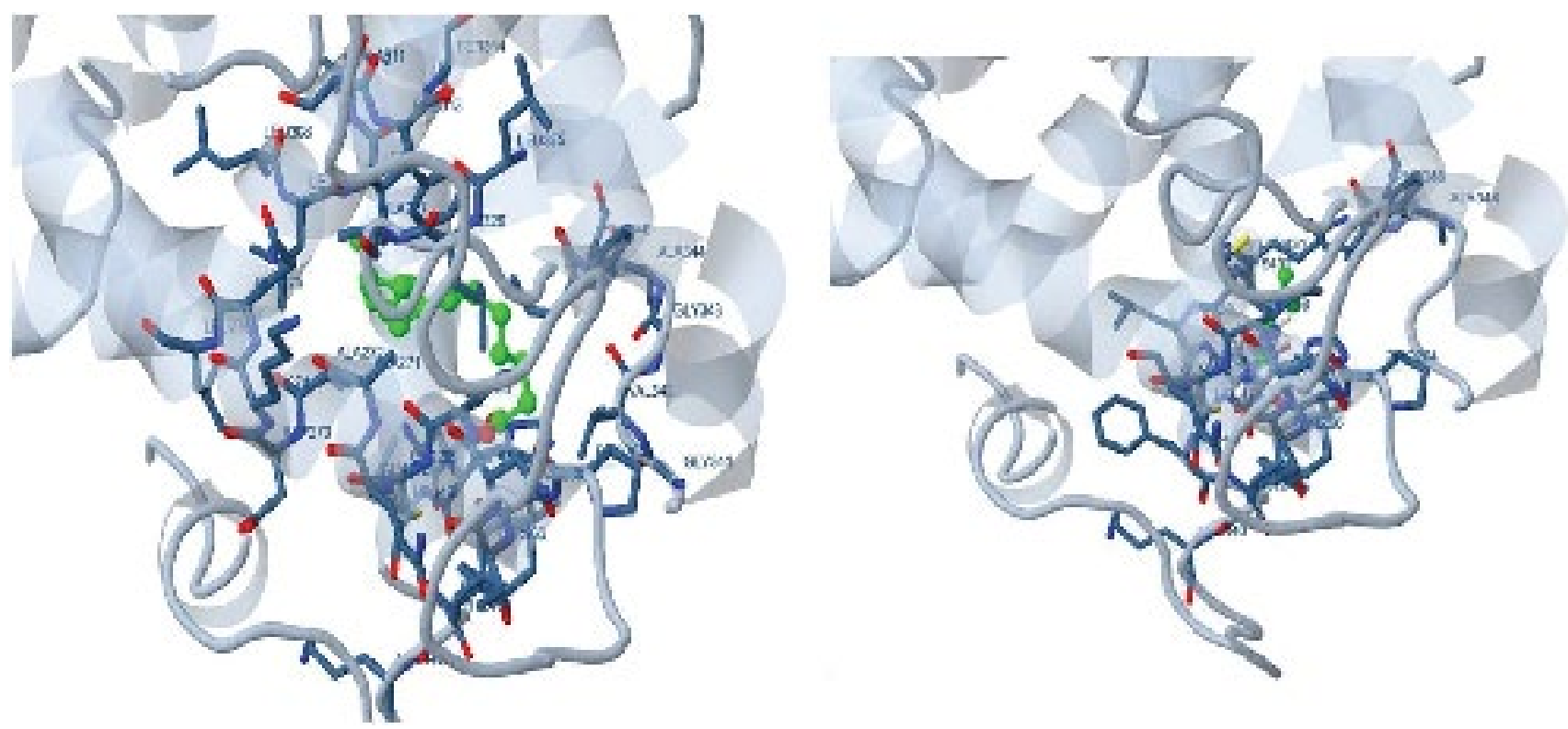

Figure 3: (i) And 3(ii) Structure of the protein-ligand complex..
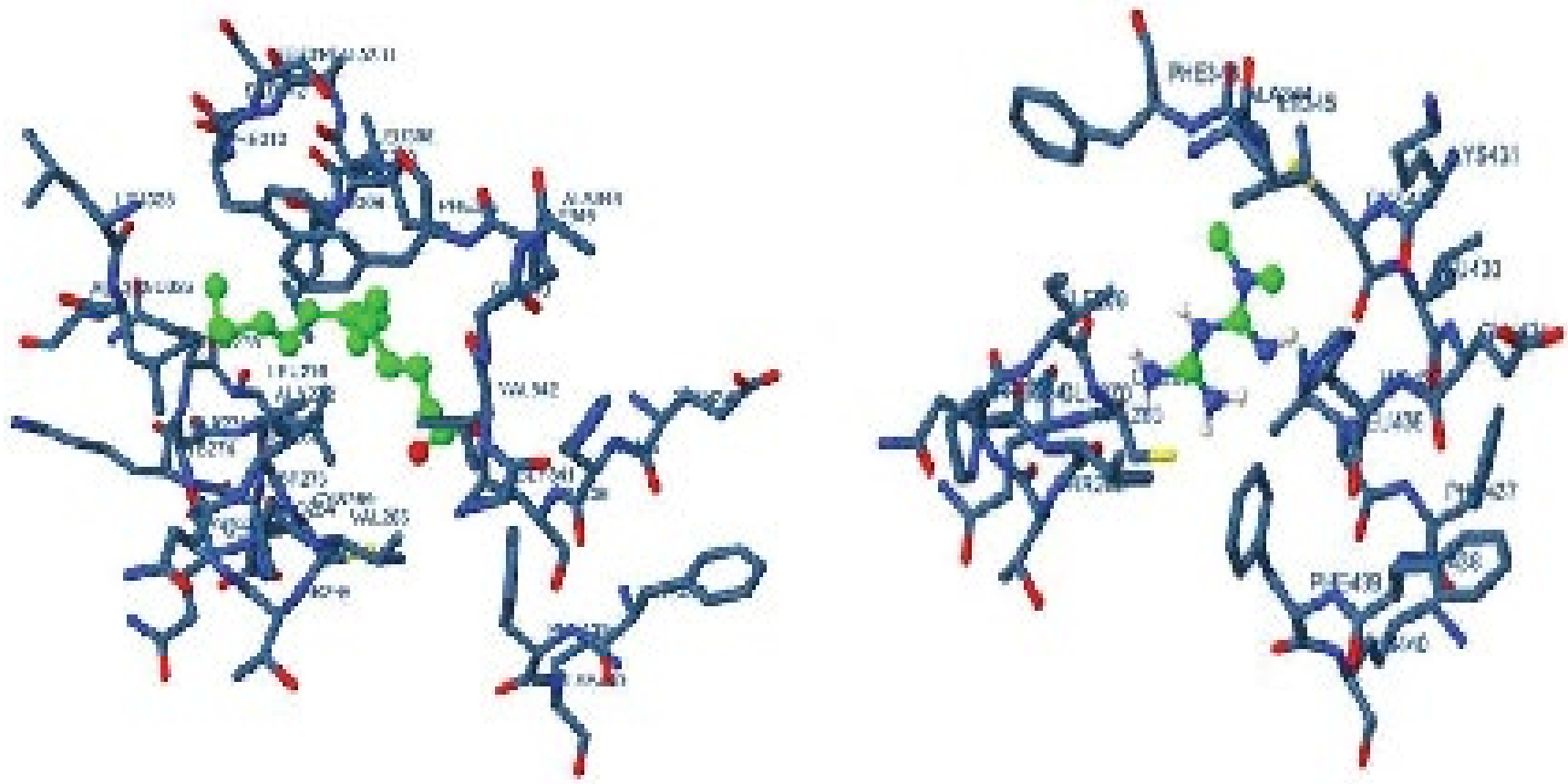

Figure 4: (i) And 4(ii) shows the position of the active site loop of the target protein.

synthetic compound. Although synthetic medicines are efficient in the emergency situations where time is the essential factor, herbal medicines are preferable in the long term treatment and prevention of the disease as they provide nutritional and immunological support also. Interaction of different Phytoconstituents in the herbs will potentiate therapeutic effects of the herb and also the mixture of two or more bioactive compounds having similar pharmacological effect may result more efficient than expected for the individual constituent. Hence, combination of potent anti-diabetic formulations from herbal source may be more beneficial. Animal studies and clinical studies are needed for further validation of the potent bioactive compound for its antidiabetic property.

\section{Acknowledgements}

The authors acknowledge UGC-UPE scholarship being availed at Karnataka University, Dharwad-580 003, Karnataka, India. 

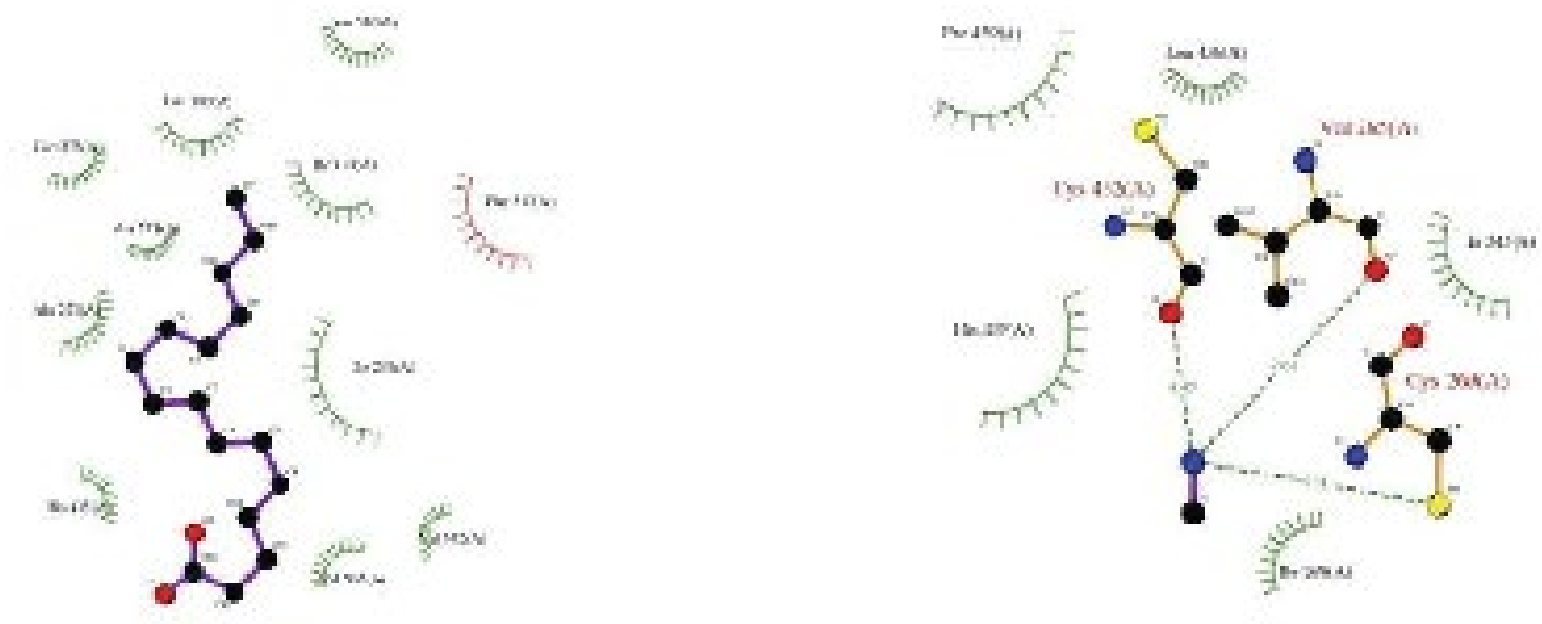

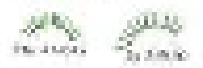

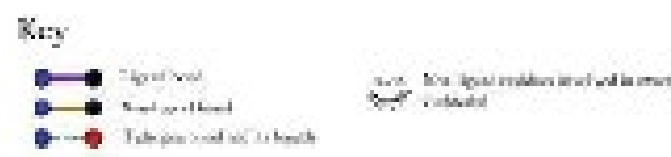

docking
Key

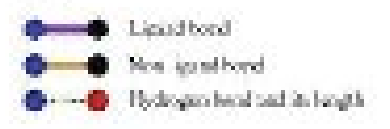

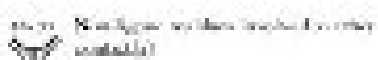

Figure 5: 2D Plot of the (i) Bioactive Compound (Hexadecanoic acid) and 5(ii) Metformin.

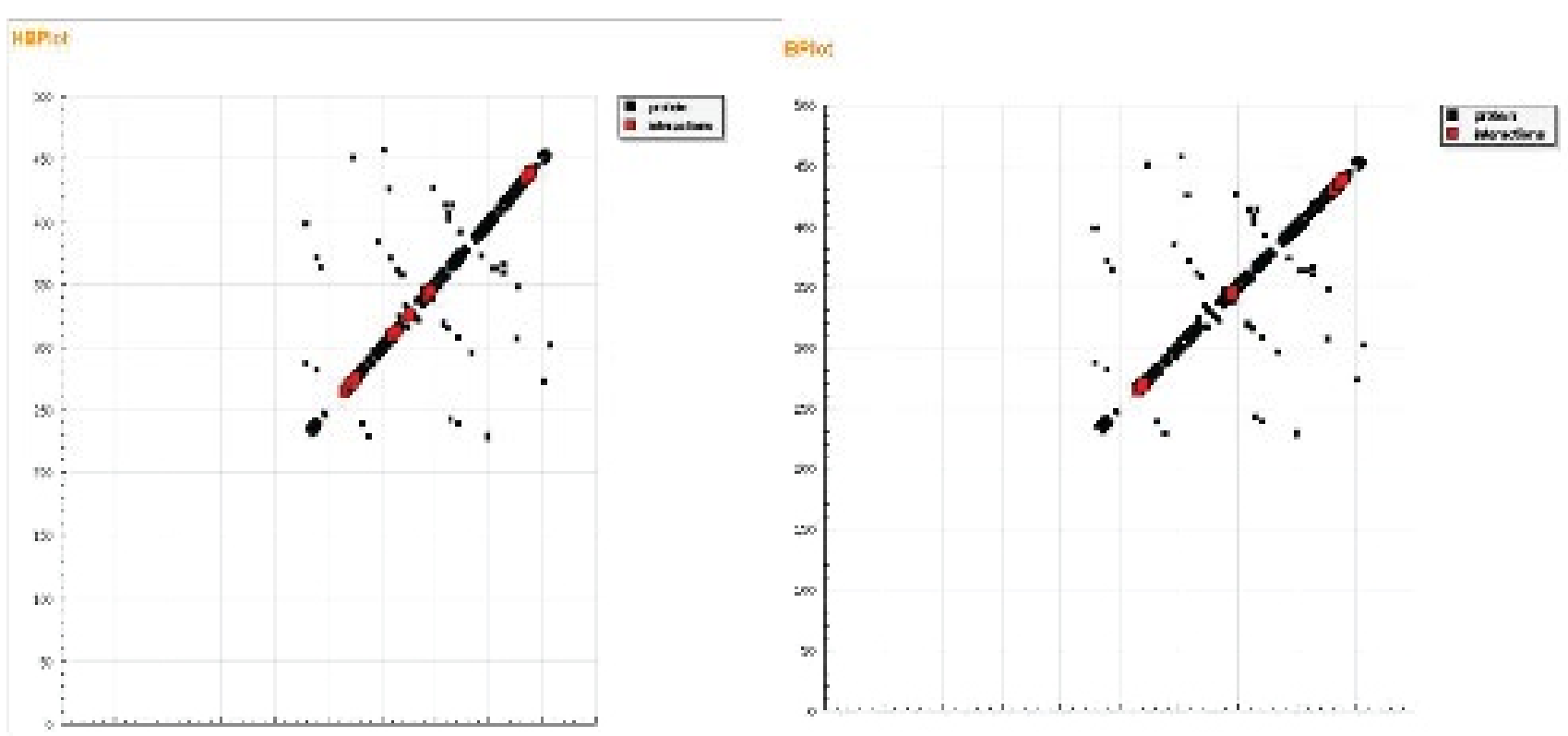

Figure 6: (i) And 6(ii) explains the Protein-Ligand interaction. 
Citation: Huttada L, Hiremath MB, D'Souza NL (2016) Enhancing the Activity of Peroxisome Proliferator- Activated Receptor's (PPAR) Activity through Natural Ligand Binding in Diabetes: Substantial Computational Approach. Nat Prod Chem Res 4: 213. doi:10.4172/2329-6836.1000213

Page 6 of 6

\begin{tabular}{|c|c|c|}
\hline $\begin{array}{c}\text { Estimated Free Energy of } \\
\text { Binding }\end{array}$ & Bioactive Compound & Metformin \\
\hline $\begin{array}{c}\text { Estimated Constant } \mathbf{k}_{\mathrm{i}} \\
\text { Inhibition }\end{array}$ & $-5.57 \mathrm{Kcal} / \mathrm{mol}$ & $-2.69 \mathrm{Kcal} / \mathrm{mol}$ \\
\hline $\begin{array}{c}\text { VdW+Hbond+desolving } \\
\text { Energy }\end{array}$ & $-9.25 \mathrm{Kcal} / \mathrm{mol}$ & $-2.59 \mathrm{Kcal} / \mathrm{mol}$ \\
\hline $\begin{array}{c}\text { Electrostatic Energy } \\
\text { Total Energy } \\
\text { Intermolecular }\end{array}$ & $0.00 \mathrm{Kcal} / \mathrm{mol}$ & $-0.10 \mathrm{Kcal} / \mathrm{mol}$ \\
\hline Frequency & $-9.25 \mathrm{Kcal} / \mathrm{mol}$ & $-2.69 \mathrm{Kcal} / \mathrm{mol}$ \\
\hline Interaction Surface & $50 \%$ & $100 \%$ \\
\hline
\end{tabular}

Table 1: The calculated docking results of the minimum energy value for the proteinligand complex of Bioactive Compound (Hexadecanoic acid) and Metformin.

\begin{tabular}{|c|c|}
\hline \multicolumn{2}{|c|}{ Interactions } \\
\hline Metformin & Hexadecanoic acid \\
\hline $265:$ VAL & $265: \mathrm{VAL}$ \\
\hline $268:$ ILE & $268:$ ILE \\
\hline $269: \mathrm{CYS}$ & $269: \mathrm{CYS}$ \\
\hline $345: \mathrm{ILE}$ & $272: \mathrm{ALA}$ \\
\hline $432: \mathrm{CYS}$ & $305: \mathrm{TRP}$ \\
\hline $435: \mathrm{HIS}$ & $309: \mathrm{EU}$ \\
\hline $436: \mathrm{LEU}$ & $310:$ ILE \\
\hline $439:$ PHE & $313: \mathrm{PHE}$ \\
\hline & $342: \mathrm{VAL}$ \\
\hline & $345:$ ILE \\
\hline & $346: \mathrm{HE}$ \\
\hline & $432: \mathrm{CYS}$ \\
\hline & $436: \mathrm{LEU}$ \\
\hline & $439: \mathrm{PHE}$ \\
\hline
\end{tabular}

Table 2: Amino acid interaction with ligand in the protein groove in the Figure 5 (i) and 5 (ii).

\begin{tabular}{|c|c|c|}
\hline Parameters & Hexadecanoic acid & Metformin \\
\hline Molecular weight & 255.416 & 130.172 \\
\hline Molpol & 30.71 & 13.00 \\
\hline Log P & 5.61 & -0.30 \\
\hline pKa & 4.95 & - \\
\hline pK & 0 & 11.60 \\
\hline Psa & 37.30 & 91.49 \\
\hline Log D & 3.57 & -3.07 \\
\hline Total charge & 0.001 & 1.651 \\
\hline Log K & -13.556 & 0.918 \\
\hline MMFF94 energy & $-0.29452 \mathrm{Kcal} / \mathrm{mol}$ & $-324.99654 \mathrm{Kcal} / \mathrm{mol}$ \\
\hline
\end{tabular}

Table 3: Predicted properties of the bioactive compound and Metformin.

\section{References}

1. Inzucchi SE, Bergenstal RM, Buse JB, Diamant M, Ferrannini E, et al. (2012) Management of hyperglycemia in type 2 diabetes: a patient-centered approach position statement of the American Diabetes Association (ADA) and the European Association for the Study of Diabetes (EASD). Diabetes care 35: 1364-1379.

2. Chang CL, Lin Y, Bartolome AP, Chen YC, Chiu SC, et al. (2013) Herbal therapies for type 2 diabetes mellitus: chemistry, biology, and potential application of selected plants and compounds. Evid Based Complement Alternat Med 2013: 378657.

3. Tiwari BK, Pandey KB, Abidi AB, Rizvi SI (2013) Therapeutic potential of Indian medicinal plants in diabetic condition. Annals of Phytomedicine 2: 37-43.
4. Modak M, Dixit P, Londhe J, Ghaskadbi S, Devasagayam TP (2007) Indian herbs and herbal drugs used for the treatment of diabetes. J Clin Biochem Nutr 40: 163-173

5. Cleveland Clinic (2016) Diabetes Mellitus: An Overview - Cleveland Clinic (Accessed 18th Feb 2016).

6. Khardori R, George T Griffing (2015) Type 2 Diabetes Mellitus (Accessed on: Oct 8,2015$)$

7. Wikipedia (2016) Diabetes Mellitus. (Accessed on 8 February 2016).

8. Wikipedia (2016) Diabetes Mellitus Type 2. (Accessed on 8 February 2016).

9. Nauck M, Frid A, Hermansen K, Shah NS, Tankova T, et al. (2009) Efficacy and safety comparison of liraglutide, glimepiride, and placebo, all in combination with metformin, in type 2 diabetes: the LEAD (liraglutide effect and action in diabetes)-2 study. Diabetes Care 32: 84-90

10. Laura GP (2011) Identification of Natural Products as Antidiabetic Agents Using Computer-Aided Drug Design Methods. Doctoral Thesis, Universitat Rovira I Virgil, Tarragona.

11. Berger JP (2005) Role of PPAR gamma, transcriptional cofactors, and adiponectin in the regulation of nutrient metabolism, adipogenesis and insulin action: view from the chair. Int J Obes (Lond) 29 Suppl 1: S3-4.

12. Berger J, Moller DE (2002) The mechanisms of action of PPARs. Annu Rev Med 53: 409-435.

13. Zoete V, Grosdidier A, Michielin O (2007) Peroxisome proliferator-activated receptor structures: ligand specificity, molecular switch and interactions with regulators. Biochimica et Biophysica Acta (BBA)-Molecular and Cell Biology of Lipids 1771: 915-925.

14. Donma MM, Donma O (2016) Promising Link between Selenium and Peroxisome Proliferator Activated Receptor Gamma in the Treatment Protocols of Obesity as well as Depression. Medical Hypotheses 89: 79-83.

15. Harborne JB (1973) Phytochemcial methods London. Chapman and Hall Ltd: 49-188.

16. Brooks BR, Bruccoleri RE, Olafson BD, States DJ, Swaminathan S, et al. (1983) CHARMM: A program for macromolecular energy, minimization, and dynamics calculations. Journal of computational chemistry 4: 187-217.

17. Tapsell LC, Hemphill I, Cobiac L, Patch CS, Sullivan DR, et al. (2006) Health benefits of herbs and spices: the past, the present, the future. Med J Aust 185 S4-24.

18. Lai PK, Roy J (2004) Antimicrobial and chemopreventive properties of herbs and spices. Curr Med Chem 11: 1451-1460.

19. Morris GM, Goodsell DS, Halliday RS, Huey R, Hart WE, et al. (1998) Automated docking using a Lamarckian genetic algorithm and an empirical binding free energy function. Journal of computational chemistry: 1639-1662.

20. Bikadi Z, Hazai E (2009) Application of the PM6 semi-empirical method to modeling proteins enhances docking accuracy of AutoDock. J Cheminform 1 : 15.

21. Solis FJ, Wets RJB (1981) Minimization by random search techniques. Mathematics of operations research: 19-30.

22. Huey R, Morris GM, Olson AJ, Goodsell DS (2007) A semiempirical free energy force field with charge-based desolvation. J Comput Chem 28: 1145-1152.

23. Morris GM, Huey R, Lindstrom W, Sanner MF, Belew RK, et al. (2009) AutoDock4 and AutoDockTools4: Automated docking with selective receptor flexibility. J Comput Chem 30: 2785-2791.

24. Singh A (2008) Phytochemicals of Gentianaceae: a review of pharmacological properties. International Journal of Pharmaceutical Sciences and Nanotechnology: 33-36.

25. Kitchen DB, Decornez H, Furr JR, Bajorath J (2004) Docking and scoring in virtual screening for drug discovery: methods and applications. Nat Rev Drug Discov 3: 935-949

26. Bikadi Z, Demko L, Hazai E (2007) Functional and structural characterization of a protein based on analysis of its hydrogen bonding network by hydrogen bonding plot. Arch Biochem Biophys 461: 225-234.

27. McDonald IK, Thornton JM (1994) Satisfying hydrogen bonding potential in proteins. J Mol Biol 238: 777-793. 